\title{
Investigations on the Effects of Different Tool Edge Geometries in the Finite Element Simulation of Machining
}

\author{
Lei Wan - Dazhong Wang* -Yayun Gao \\ Shanghai University of Engineering Science, College of Mechanical Engineering, China
}

This work focuses on the effects of cutting edge geometries on dead metal zone formation, as well as stress and temperature distributions in orthogonal cutting of P20 material using finite element method (FEM) simulation with sharp, chamfered, double chamfered and blunt tools. The cutting process is simulated with Arbitrary Lagrangian-Eulerian (ALE) approach in ABAQUS/Explicit. The simulation results suggest that the tool edge geometry influences the shape of dead metal zone considerably, while having little influence on the chip formation. An analysis of thermo-mechanical coupling was also conducted, and the results show that the stress distribution is affected by the temperature distribution and cutting speed because of the thermal softening effect and the strain rate hardening. A common analytical model is introduced to predict the residual stress, and equivalent Mises residual stresses are all calculated with four different tools to suggest that the tool edge geometry has a significant effect on the residual stress. The experiments are conducted using a CNC with former four kinds of tools at a speed of 480 $\mathrm{m} / \mathrm{min}$, and the residual stresses beneath the machined surface were measured with X-ray diffraction and electro-polishing techniques, and a chamfer tool at three different cutting speeds $(250,600$ and $1000 \mathrm{~m} / \mathrm{min})$ to obtain the forces. The machining forces in both the cutting and thrust directions increases as the chamfer angle increases and decreases as the cutting speed increases.

Keywords: tool edge geometry, coupled thermo-mechanical analysis, finite element method, dead metal zone, residual stress

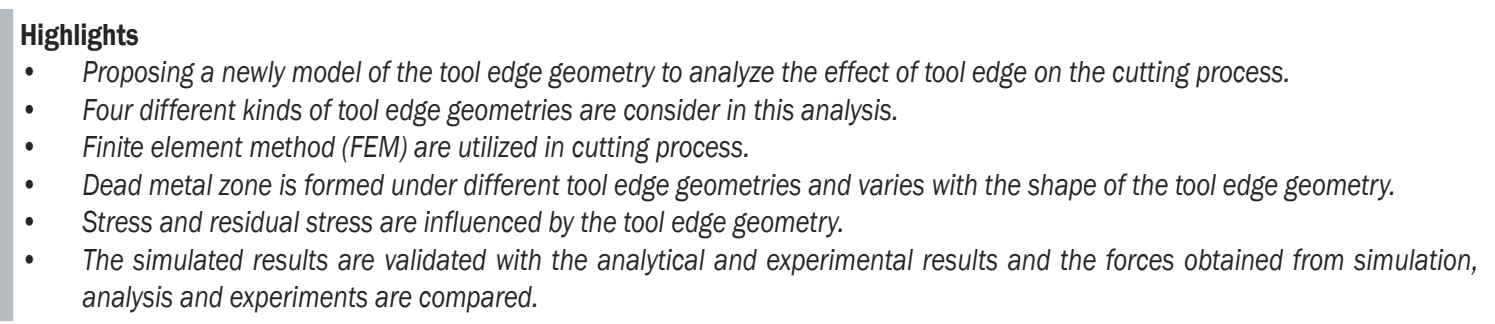

\section{INTRODUCTION}

Metal cutting is always considered to be one of the most complicated manufacturing processes for different materials. In order to attain sufficiently high production rates at minimal cost, optimization of cutting tool geometry is necessary. Moreover, the cutting process is greatly influenced by the cutting conditions, such as cutting velocity, chip thickness, and feed rate, as well as the tool geometry. The design of tool edge geometry influences process parameters, such as the shape of deformation zones, stresses on the chip and machined surface and the cutting forces mentioned in Fig. 1 [1]. These effects in turn affect the changes in chip flow and machined surface integrity. The modification of the tool edge geometry is referred to edge preparation. Fig. 2 illustrates four major types of edge preparation design used in most commercial cutting inserts: sharp edge; hone edge; T-land/chamfer edge and double chamfer edge. Moreover, Regions 1, 2, 3 are the missing regions of blunt, double chamfer and chamfer tools compared with the sharp tool, respectively.

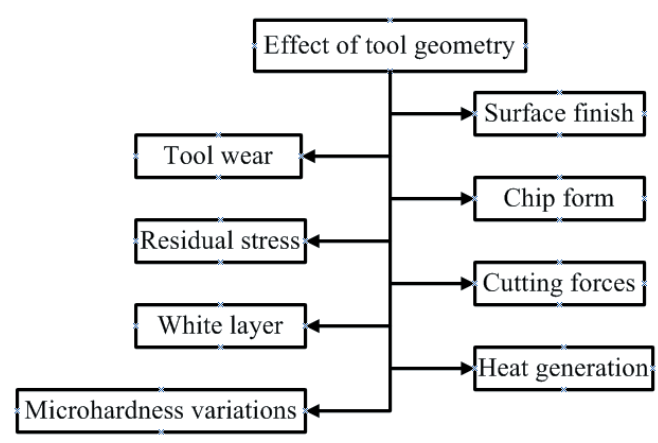

Fig. 1. Effect of tool geometry on performance parameters in turning

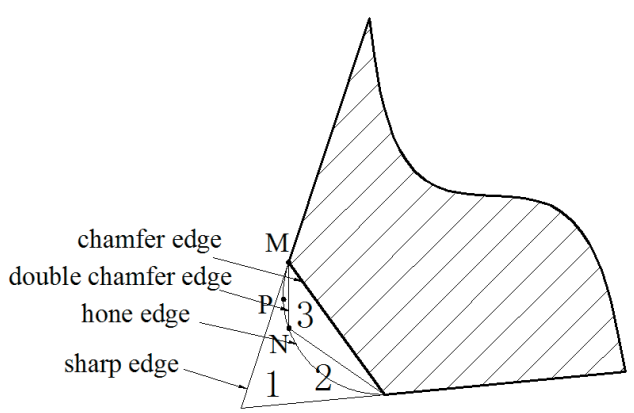

Fig. 2. Typical cutting edge preparation 
Much research has been conducted about how the edge geometry of tools influenced the cutting process, analytically and experimentally [2]. Experimental results of a study done by El-Wardany et al. [3] show that for a sharp tool (compared with other geometries) the magnitude of the residual stress on the machined surface and the penetration depth of the stressed layer were reduced as cutting speed increased, while there is an opposite trend for the hone tools. Based on the large numbers of experiments, which were conducted with $\mathrm{CBN}$ tools for carburized hardened steel (600 to $720 \mathrm{HV}$ ) under both continuous and interrupted cutting conditions, Shintani et al. [4] and [5] analysed the effect of tool geometry on the cutting performance. They proposed that the optimum tool geometry for continuous cutting be specified as having a negative chamfer angle of $35^{\circ}$ and a nose radius of $0.8 \mathrm{~mm}$. Matsumoto et al. [6] investigated four different tool edge geometries (sharp, honed, single chamfered and double chamfered) on residual stress in precision hard turning, and concluded that tool edge geometry is the dominant factor determining the residual stress profile, and the residual stress with the honed and chamfer tool on the machined surface became more compressive. In recent decades, with the improvement of the computer technology, the finite element method (FEM) and numerical simulation have been increasingly used to study the machining process. Kountanya et al. [7] studied the effect of tool geometry and cutting conditions on experimental and simulated chip formation. Some observations can be obtained to illustrate that the machining forces increase as the tool edge radius increases, and the change of edge radius has little influence on chip morphology. Shatla et al. [8] applied the Lagrangian FEM to simulate the cutting process of $\mathrm{H} 13$ tool steel to investigate the influence of edge preparation (hone/chamfer) on tool temperature and stress. The simulation results showed that an increasing tool edge radius alters the distribution of temperature in the different kinds of tools, while the experiment results also indicated that the cutting force increases as the tool edge radius increases.

When studying the influence of the tool geometry on the metal cutting process, one of the main points of interest is the existence of the dead metal zone under the chamfer or around the edge radius of the tool. The dead metal zone is trapped under the chamfer and almost entirely fills the chamfer, which acts as the effective cutting edge of the tool, thus protecting the tool surface from wear under various kinds of heavy cutting conditions. The drawbacks of cutting with a dead metal zone on the chamfered edge are that the forces on the tool increased, and the simulation results may be inaccurate due to the varying dead zone during cutting. Experimental and analytical studies discussed in [9] to [11] concluded that a dead metal zone is mostly dependent on the geometry of the chamfered part rather than the cutting conditions. Meanwhile, the dead zone is also formed with the blunt tool in the same way as for the chamfered tool, which is in good agreement with the results of assumption of the formation of BUE discussed by Waldorf et al. [12] for large-radius tools. The region of the missing zone determines the region of the dead zone. The surface finishing may depend greatly on the size and the shape of the dead zone. Al-Athel and Gadala [13] proposed a new volume of solid (VOS) method to simulate the metal cutting process with four different geometries tools. The simulation results suggest that the tool geometries have great influence on the formation of the dead metal zone, but little effect on the distribution of stress. Jacobson et al. [14] conclude that various kinds of dead zones near the tip of the tool may occassionaly become unstable in form and size, and sometimes crack partially or even entirely, resulting in deposits on the tool surface.

From this background information, it can be determined that the above mentioned authors have the common view that the tool edge geometries significantly influence dead metal zone formation and residual stress distribution, but not the chip formation and the stress distribution. Aiming at these hypotheses, this paper presents a numerical analysis of a continuous chip formation process based on the finite element method for a better understanding of dead metal formation and chip formation. The effects of four common kinds of tool edge geometries on the formation of the dead metal zone and how they affect the distributions of stress and temperature have been studied.

\section{NUMERICAL SIMULATION}

\subsection{Numerical Approach}

Numerical simulation of the cutting process can provide detailed results for process variables, such as stress, strain, strain rate, temperature, that are extremely difficult to measure with current technology. Owing to advanced technology and computer power, an alternative approach called the arbitrary Lagrangian-Eulerian (ALE) approach [13], which could combine the advantages of Lagrangian and Eulerian approaches, eliminating mesh distortion in Eulerian formulation and modeling the 
unconstrained flow of the chip in Lagrangian approach was applied in this research. The ALE approach is proved to be viable once a proper mesh motion scheme been implemented, Fig. 3 [15]. The simulation results are compared with the numerical work done by Movahhedy et al. [16], who simulated the cutting process via the ALE approach with four different tools whose rake angles were $0^{\circ}$. The ALE model (shown in Fig. 4) developed by Muñoz-Sánchez et al. [17] allows the material to flow across an internal Eulerian zone surrounding the tool tip by using sliding Lagrangian and Eulerian contours. This method can avoid extreme mesh distortion and allows the simulation of a long machining time. The model is divided into four zones, allowing mesh motion or material flow across the fixed mesh. Zones 1 to 3 combine Lagrangian/ Eulerian boundaries with sliding boundaries where the material can flow tangential to the contour but not go across this boundary. Eulerian boundaries located at the entrance of Zone 1 and in Zone 2 can avoid the mesh distortion that is commonly revealed as the calculation advances. Zone 4 allows the material to flow across this region (which is a Eulerian region) with the mesh fixed. The main advantage of this technique is that it can avoid the extreme distortion in the region surrounding the tool tip. Therefore, it can be used to simulate a large machined surface in this paper.

\subsection{Boundary Conditions and Material Properties}

Fig. 5 shows the schematic of the metal cutting process for a general case with a chamfer tool. The cutting force and thrust force are respectively defined by $F_{c}$ and $F_{t} . F_{s}$ and $F_{n}$ represent the forces acting

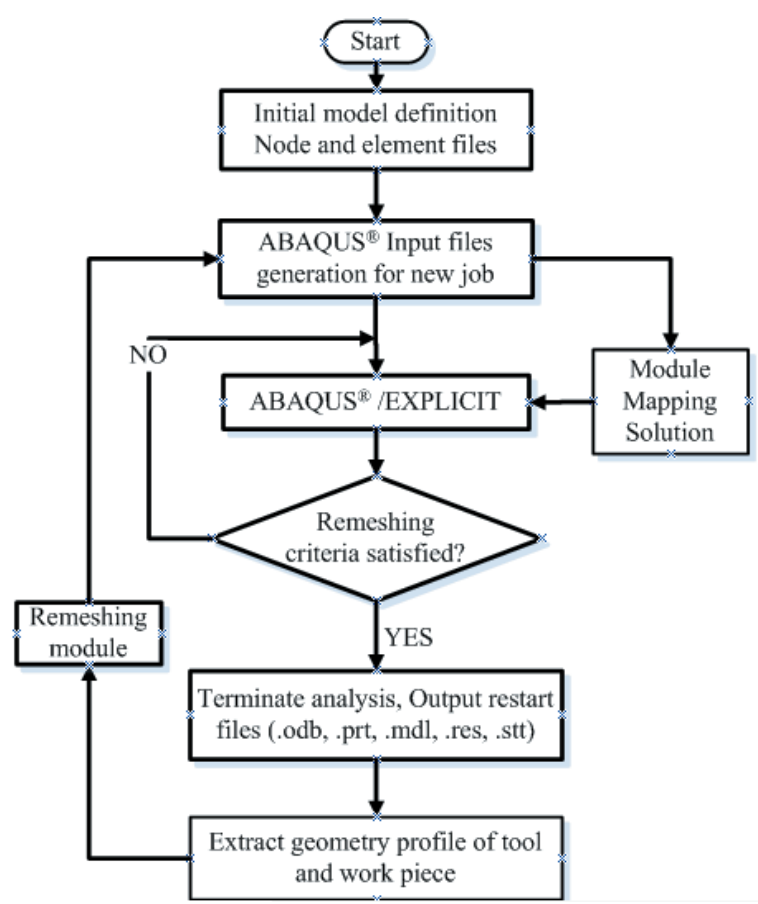

Fig. 3. Overall simulation approach

along and normal to the shear plane, respectively. The main rake angle and chamfer angles are defined as $\alpha_{0}$ and $\alpha_{1}$. The length of the chamfered part is defined as $b_{c f}$, material flow $\left(V_{c}\right)$ splits into two parts at the front of the cutting edge, one part shapes the chip at speed of $V_{a}$, the other forms the machined surface at speed of $V_{b}$. Three deformation zones are shown in the graph.

The metal cutting process is so complicated that the finite element model should be simplified, and some assumptions established as well. The assumptions of finite element model are:

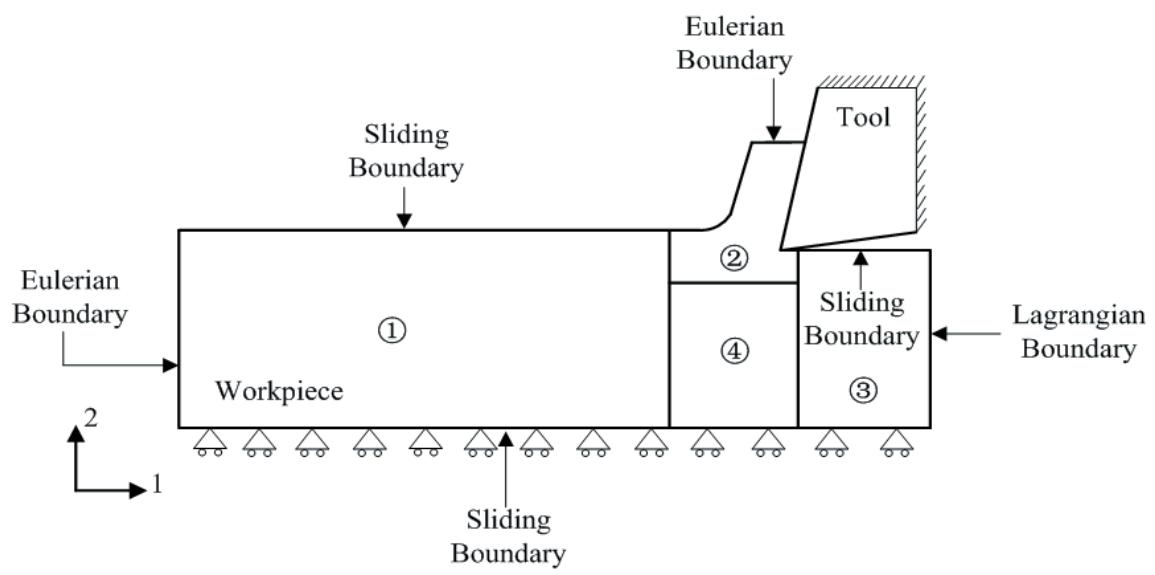

Fig. 4. Implementation of boundary condition and type of contour in the model; regions 1 to 3 combine sliding and Lagrangian/Eulerian boundaries; region 4 combines Eulerian boundaries 
1. The workpiece material is isotropic.

2. The workpiece material accords with Von Mises yield criteria.

3. Machine tool bed and fixture are both rigid in the cutting process.

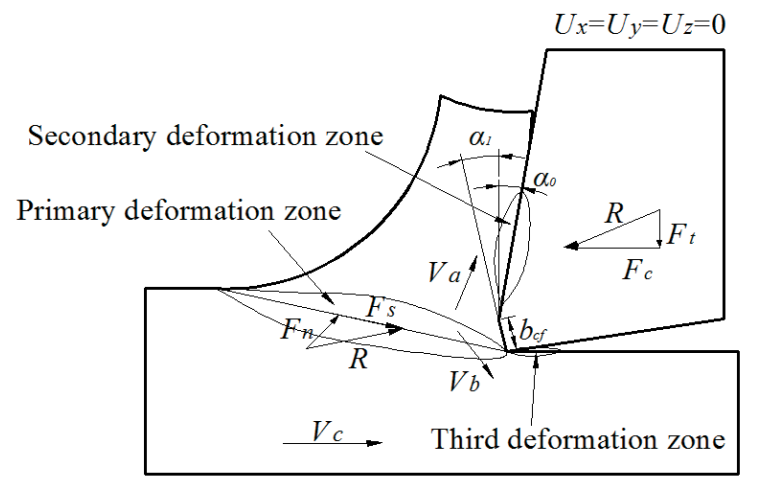

Fig. 5. Schematic view of metal cutting process with double chamfered tool

In the actual metal turning process, cutting width is much larger than the back engagement of the cutting edge, so the chip deformation, which is perpendicular to the cross-section of tool edge, is approximately same along the tool edge direction, which is called the plane strain state. Therefore, the metal cutting process can be regarded as a two-dimensional orthogonal cutting model when simulating the cutting process by using the FEM.

In this paper, basic geometry and boundary conditions of the numerical model are shown in Fig. 4 , and a plain strain condition is assumed. The tool is fixed and cutting speed is applied to the workpiece to obtain the velocity field of the workpiece material, the rake angle is $10^{\circ}$, and the clearance angle is $7^{\circ}$. Material properties for the workpiece and tool are presented in Table 1.

Table 1. Material properties for the workpiece and tool

\begin{tabular}{lcc}
\hline Material properties & Workpiece & Tool \\
\hline Material & P20 & Carbide tool \\
\hline Young's modulus [GPa] & 210 & 530 \\
\hline Poisson's ratio & 0.3 & 0.3 \\
\hline Conductivity [ $\left.\mathrm{W} \mathrm{m}^{-1}{ }^{\circ} \mathrm{C}^{-1}\right]$ & 51.5 & 120 \\
\hline Specific heat $\left[\mathrm{J} \mathrm{kg}^{-1}{ }^{\circ} \mathrm{C}^{-1}\right]$ & 470 & 343.3 \\
\hline Thermal expansion coefficient $\left[{ }^{\circ} \mathrm{C}^{-1}\right]$ & $1.4 \times 10^{-6}$ & $5.2 \times 10^{-6}$ \\
\hline
\end{tabular}

In order to correctly simulate the cutting process with different tool geometry, it is necessary to introduce a material flow stress model to describe the material behaviour. The model is obtained from [18], which considers high temperature and high strain rate, usually presented with the equation below:

$$
\bar{\sigma}=\left(A+B(\bar{\varepsilon})^{n}\right)\left(1+C \ln \left(\frac{\dot{\bar{\varepsilon}}}{\dot{\overline{\varepsilon_{0}}}}\right)\right)\left(1-\left(T^{*}\right)^{m}\right)
$$

with the $T^{*}$ is defined as:

$$
T^{*}=\frac{T-T_{r}}{T_{m}-T_{r}}
$$

where $\bar{\sigma}$ is the equivalent flow stress, $\bar{\varepsilon}$ the equivalent plastic strain, $\dot{\bar{\varepsilon}}$ the equivalent plastic strain rate, $\dot{\overline{\varepsilon_{0}}}$ the reference strain rate, which equals $1 \mathrm{~s}^{-1}$. The material characteristics are defined by the thermal softening coefficient $m$, the strain hardening exponent $n$, and constants adopted from [13], which are listed in Table 2. $T_{m}$ and $T_{r}$ are the material melting temperature $\left(1480{ }^{\circ} \mathrm{C}\right)$ and reference ambient temperature $\left(20^{\circ} \mathrm{C}\right)$, respectively.

Table 2. J-C parameters for $P 20$

\begin{tabular}{ccccc}
\hline $\mathrm{A}[\mathrm{MPa}]$ & $\mathrm{B}[\mathrm{MPa}]$ & $\mathrm{C}$ & $n$ & $m$ \\
\hline 178.5 & 462.4 & 0.0438 & 0.169 & 0.666 \\
\hline
\end{tabular}

\subsection{Frictional Model}

Whether the cutting simulation results are accurate and reasonable, to a large degree depends on the foundation of the frictional model; therefore, it is vitally important to choose a reasonable friction model. The rake face is divided into two workspaces, the sticking zone and the sliding zone, which is illustrated in Fig. 6.

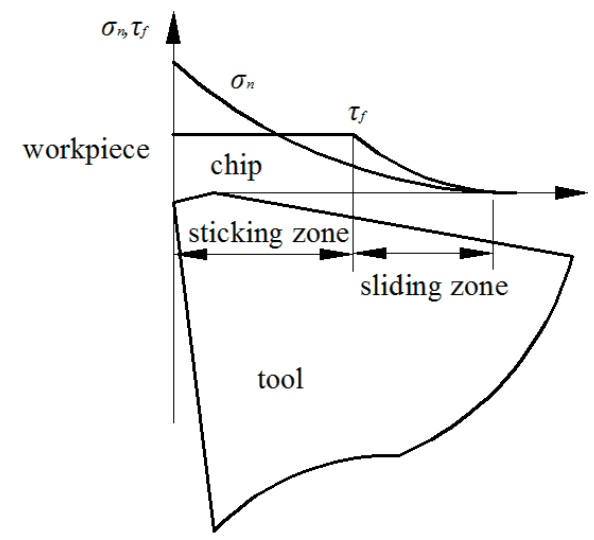

Fig. 6. Curves representing normal and frictional stress distributions on the tool rake face

Alvarez et al. [19] studied the effect of four different constitutive models and three friction 
coefficients $(0.4,0.6$ and 0.8$)$ on the simulation results. They conclude that the friction factor depends on the constitutive equations and their parameters, and its election is subject to the experiment results. Thus, when considering machining simulations of P20, a moderate friction coefficient (0.4) is used in this study and friction at the tool-chip interface is controlled by a Coulomb friction model which is expressed by the following relations:

$$
\begin{aligned}
\tau & =\mu \sigma_{n}, \text { if } \mu \sigma_{n}<\bar{m} \frac{\sigma_{0}}{\sqrt{3}}, \text { (the sliding zone) } \\
\tau & =\bar{m} \frac{\sigma_{0}}{\sqrt{3}}, \text { if } \mu \sigma_{n}<\bar{m} \frac{\sigma_{0}}{\sqrt{3}}, \text { (the sticking zone) }
\end{aligned}
$$

The shear stress $(\tau)$ is either expressed by the product of Coulomb friction coefficient $(\mu)$ with normal stress $\left(\sigma_{n}\right)$ or by a fraction $(\bar{m})$ of permissible shear stress of the workpiece material.

\section{EXPERIMENTAL WORK}

In this work, a disk made of P20 mould steel was turned in orthogonal mode on a CNC turning centre, and the cutting force and chip thickness were measured in each case. In the first set of tests, blank carbide tools of ISO S10 class were used with sharp and different chamfer angles $\alpha_{1}$ and lengths $b_{c f}$, as listed in Table 3 . The primary rake angle of the tool was $10^{\circ}$ in all cases. A chip load of $0.1 \mathrm{~mm}$ and a cutting speed of $480 \mathrm{~m} / \mathrm{min}$ were adopted. In the second set of tests, a chamfer tool with a primary rake angle of $10^{\circ}$ and a chamfer angle of $-25^{\circ}$ and a chamfer length of $0.1 \mathrm{~mm}$ were used to cut P20 disks at three different cutting speeds of 250,600 and 1000 $\mathrm{m} / \mathrm{min}$. The uncut chip thickness in this set was 0.06 $\mathrm{mm}$. The produced chip was continuous in all cases.

Table 3. Tool edge geometry in cutting tests with carbide tools

\begin{tabular}{cccc}
\hline Case & $\alpha_{0}\left[^{\circ}\right]$ & $\alpha_{1}\left[^{\circ}\right]$ & $b_{c f}[\mathrm{~mm}]$ \\
\hline 1 & 10 & 0 & - \\
\hline 2 & 10 & -10 & 0.0902 \\
\hline 3 & 10 & -25 & 0.0841 \\
\hline 4 & 10 & -35 & 0.0863 \\
\hline
\end{tabular}

The measurements of residual stress were conducted with an X-ray diffraction technique, and the measurement conditions used in this study are listed in Table 4 [20]. The measurements of residual stress were performed by using a ' $\sin \phi$ ' method. An electro-polishing technique was utilized to determine the residual stress beneath the machined surface, and the maximum electro-polished depth from the machined surface is over $150 \mu \mathrm{m}$.

Table 4. Conditions of $X$-ray diffraction

\begin{tabular}{ll}
\hline Characteristic X-ray & $\mathrm{Cr} \mathrm{K \alpha}$ \\
Diffraction plane & $(211)$ \\
Diffraction angle & $156.08^{\circ}$ \\
Tube voltage & $30 \mathrm{kV}$ \\
Tube current & $10 \mathrm{~mA}$ \\
Divergent angle & $1.0^{\circ}$ \\
Step angle & $0.5^{\circ}$ \\
Fixed time & $0.4 \mathrm{~s} \mathrm{per} \mathrm{step}$ \\
Irradiated area & $10 \times 20 \mathrm{~mm}$ \\
Stress constant & $-297.23 \mathrm{MPa} /{ }^{\circ}$ \\
\hline
\end{tabular}

\section{RESULTS AND DISCUSSION}

\subsection{The Existence of the Dead Metal Zone}

Fig. 7 shows the velocity profile of the material under the same cutting condition with different edge geometry tools by ALE. It is obvious for the sharp tool that there is nearly no metal dead zone except for a small region where the material flow slows down because of the block of the tool corner. However, for the chamfered tool, it is clear that part of the material is trapped under the chamfer corner, which presents low speed and acts as the missing tool nose. It is much clearer that the tool of the $-25^{\circ}$ chamfer angle has the largest dead zone in comparison to the other three tools because of its larger missing region, to indicate that the zone is dependent on the tool geometry. As for the blunt tool, a dead metal zone presents to a lesser extent, because the fillet of the tool allows a smoother flow of the material along the surface.

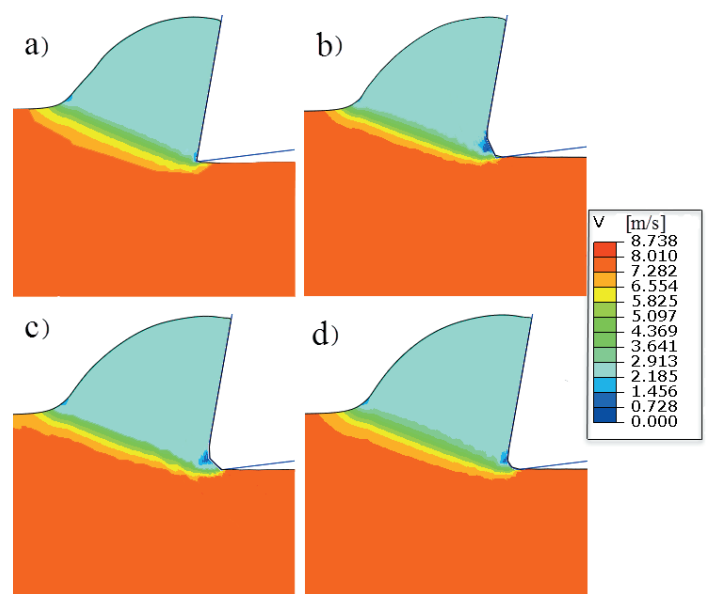

Fig. 7. Velocity fields for; a) a sharp tool, b) $25^{\circ}$ chamfered tool, c) double chamfered tool, and d) blunt tool 
The simulation results are compared with the simulations conducted by Movahhedy et al. [16] to discuss the position of the dead metal zone. The comparison illustrates that the rake angle affects the position of the dead zone significantly. It can be found that the positive rake angle will result in a shifting up of the dead zone, because the positive angle allows the material to follow past quickly along the rake face and the dead zone does not fill the missing region completely under the chamfer. It also can be found in these figures that the tips of the tools in these simulations have already intruded into the mesh of material at different extents because of the quality of the mesh. It can be solved by concentrating the meshes while the computational time will cost much more.

\subsection{The Distributions of Effective Stress and Temperature}

The properties of workpiece material at elevated temperatures and high strain rates while cutting are very different than that is not under cutting. The curves of stress-strain are predicted according to the JC model, as shown in Fig. 8, at strain rate $\dot{\varepsilon}=5000 \mathrm{~s}^{-1}$ and temperature $T=800{ }^{\circ} \mathrm{C}$. These curves reveal many important properties of the material P20. The material has a significant property of strain hardening in that the flow stress increases as the strain increases slowly. From the left figure, the quite obvious that temperature sensitivity can be found in the material so that the flow stress decreases quickly as the temperature increases, which is called temperature softening effect. Moreover, from the right one, a distinct strain rate sensitivity of the material is shown so that the flow stress increases as the strain rate increases, which can be called the strain rate hardening effect. With the strain rate growing (from 1,000 to $10,000 \mathrm{~s}^{-1}$ ), the increment of flow stress reduces slowly, causing the strain rate sensitivity of material to decrease, which is because the effect of temperature softening is stronger than strain rate hardening at high speed deformation when the temperature increases, decreasing the level of increment of flow stress.

Figs. 9 and 10 show the predicted distributions of effective stress and temperature with the four different tools during the metal cutting process, respectively. Although the chip is formed with different kinds of tool geometries, the distribution of the stress in the chip is almost the same. It is obvious for the shear zones in these contours that possess the highest stress values that extend from the beginning of the chip bight at the free surface to the edge of the tool or the dead metal zone. Moreover, the highest stress comes from the severe and rapid deformation in the primary zone during the cutting process. From Fig. 9, it can be seen that the distribution of the active stress along the rake face of the tool is highly concentrated in the chip in all cases, and the values are all minuscule. This is due to temperature softening effect which refers to the effective stress decreasing quickly as the temperature increases. The temperature in the friction region is extremely high (Fig. 10) due to the friction between chip and tool, which significantly affects the values of the stress (Fig. 9) in this region. Considering the effect of the temperature on tools, it can been found in Fig. 10 that the highest temperature zones are concentrated in the sliding zone and flank surface, because these two regions experienced the severest friction during cutting. Comparing the temperature distributions of these tools, a fine view can be obtained that the edges of the tools possess extremely high temperatures, suggesting that this region is the first to be worn out. Meanwhile, it can be found that several voids and non-smooth surfaces of the machined material exist in vicinity of the tool edge because of the relatively
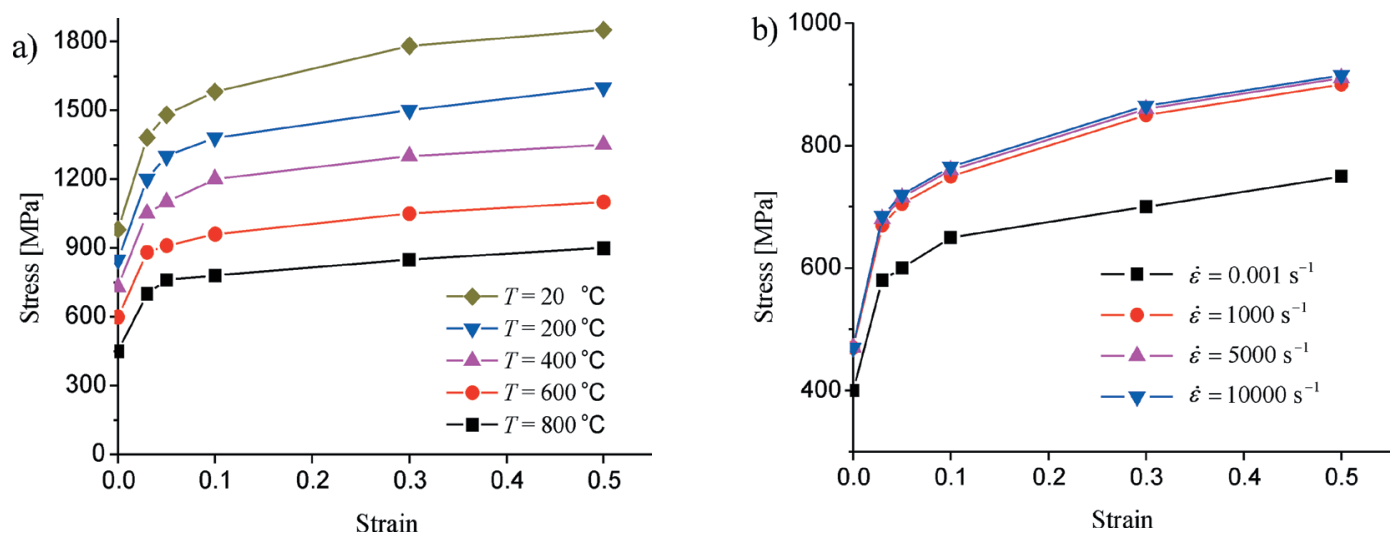

Fig. 8. Predicting stress-strain curves of JC model; a) at strain rate $\dot{\varepsilon}=5000 \mathrm{~s}^{-1}$, and b) at temperature $T=800{ }^{\circ} \mathrm{C}$ 

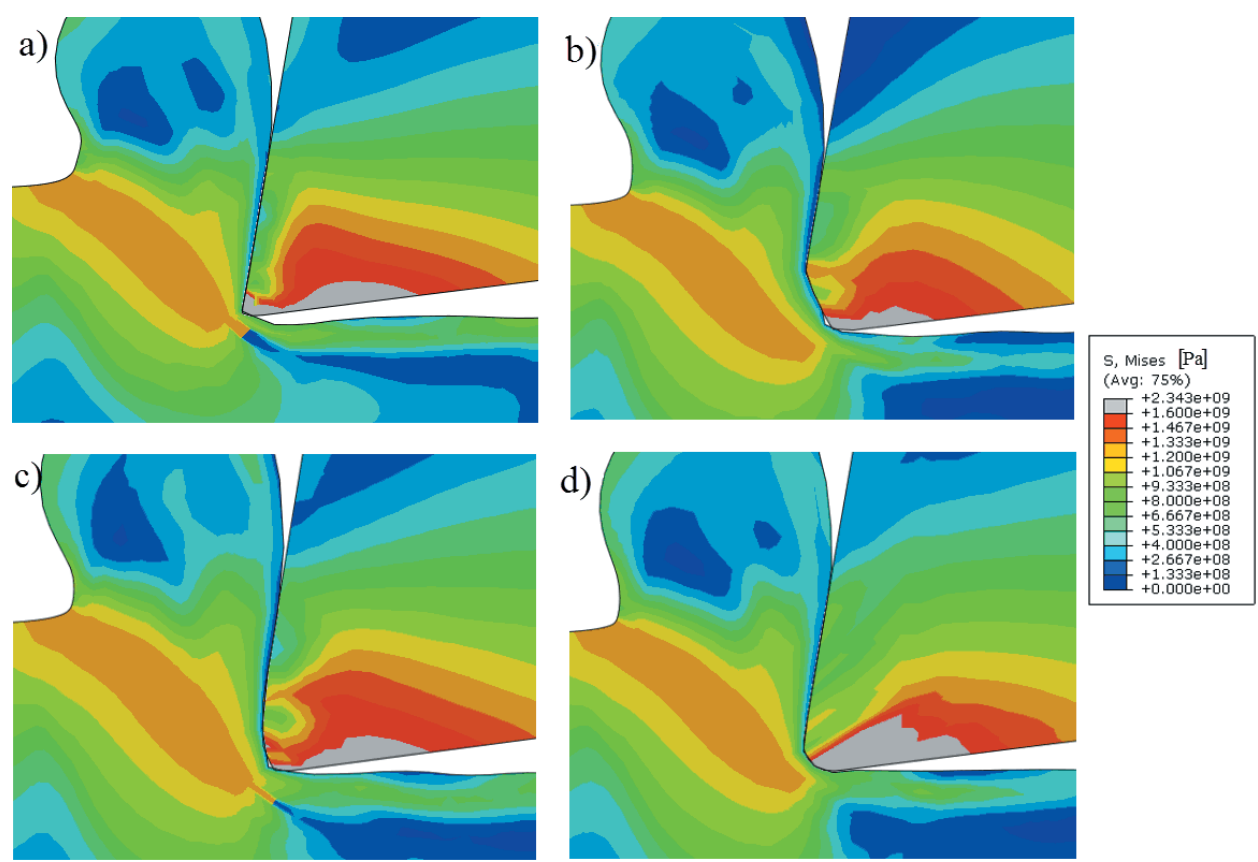

Fig. 9. Distributions of stress for a) a sharp tool, b) a tool with $25^{\circ}$ chamfer angle, c) a double chamfer tool, and d) a blunt tool
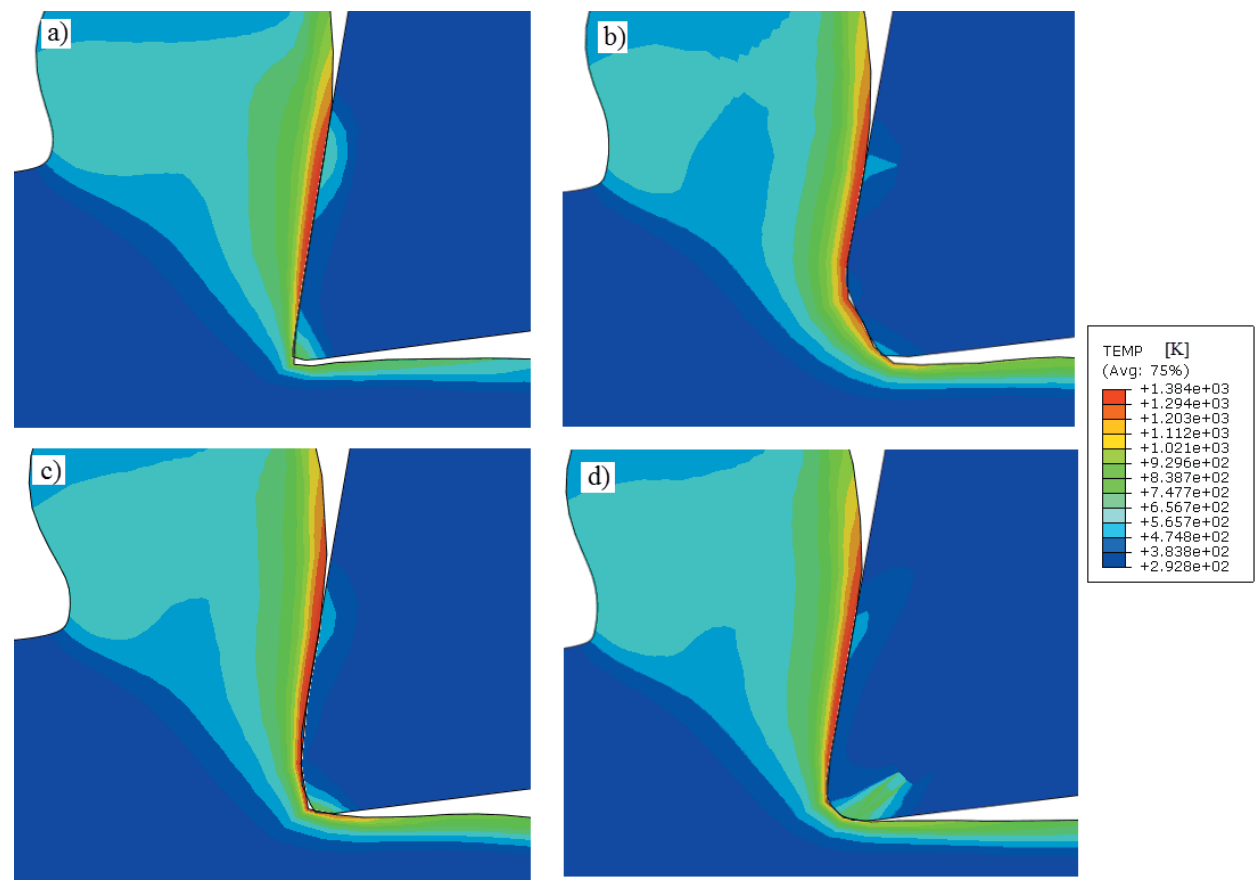

Fig. 10. Distributions of temperature for a) a sharp tool, b) a tool with $-25^{\circ}$ chamfer angle, c) a double chamfer tool, and d) a blunt tool

coarse finite element mesh used in these regions as well as the large deformation of workpiece material during the cutting process. Although finer meshing will result in more accurate simulation results, the computing time will increase significantly due to the re-meshing process. Moreover, after a relatively fine meshing, more grids will not work, but the time still increases.
Therefore, a balance between the accurate results and the computing time should be made. In this paper, the balance is made with a relatively fine meshing, considering the computing time. The voids and nonsmooth surfaces of the machined material suggest that the results obtained from these simulations will have some differences with the experimental results. 


\subsection{The Effect of Tool Geometries on the Distribution of Residual Stress}

The modelling approach is established on the material stagnation ahead of the cutting edge and the ploughed depth, as shown in Fig. 11. Material flows toward the cutting edge and splits into upward and downward streams at the stagnation in the vicinity of the point P. As for the chamfer and double chamfer tools, the apex of the tool edge (the $\mathrm{M}$ of the chamfer tool and the $\mathrm{N}$ of the double chamfer tool) is the point $\mathrm{P}$, as shown in Fig. 2. Ploughed depth $t$ is located between the ideal material separation line BD and the actual separation line PC. This part of uncut chip thickness becomes squashed by the cutting edge and contains residual stress. Moreover, the ploughed depth $t$ depends on cutting edge geometry and determines the distribution of residual stress. Therefore, a larger radius contributes to the shifting up of the point $\mathrm{P}$, thus enlarging the ploughed depth $t$. The residual stresses were obtained in the circumferential direction S11 (parallel to the cutting direction) and in the radial direction S33 (parallel to the feed direction) at the

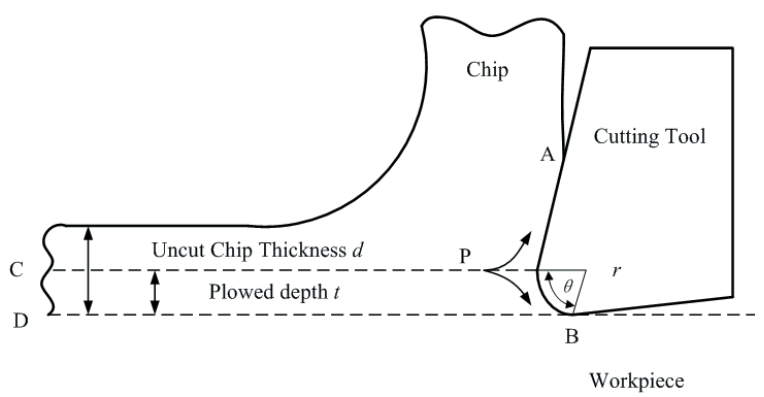

Fig. 11. Schematic of ploughed depth and material stagnation during the cutting process

model points, which are at distance 10 times the chip thickness behind the tool, as shown in Fig. 12. The shape of the profiles is almost same with four kinds of different tools in the circumferential (S11) and radial (S33) directions that residual stresses are tensile on the surface then rapidly shift to be compressive and finally stabilize at an approximate non-stress value. Dogra et al. [1] concluded that the effect of the chamfer is equivalent to the increasing hone radius to help increase compressive residual stress but less than
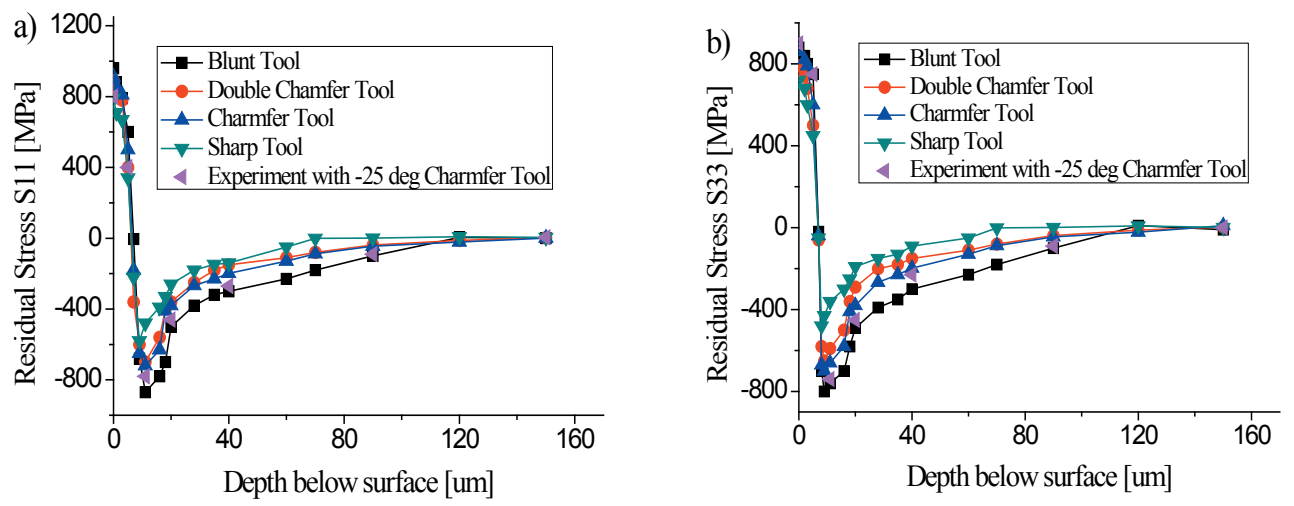

Fig. 12. The effect of different tools on residual stresses of machined P20 in the a) circumferential (S11) and b) radial (S33) directions with $V=480 \mathrm{~m} / \mathrm{min}$ and uncut chip thickness $0.1 \mathrm{~mm}$ and experimental result with $-25^{\circ}$ chamfer tool
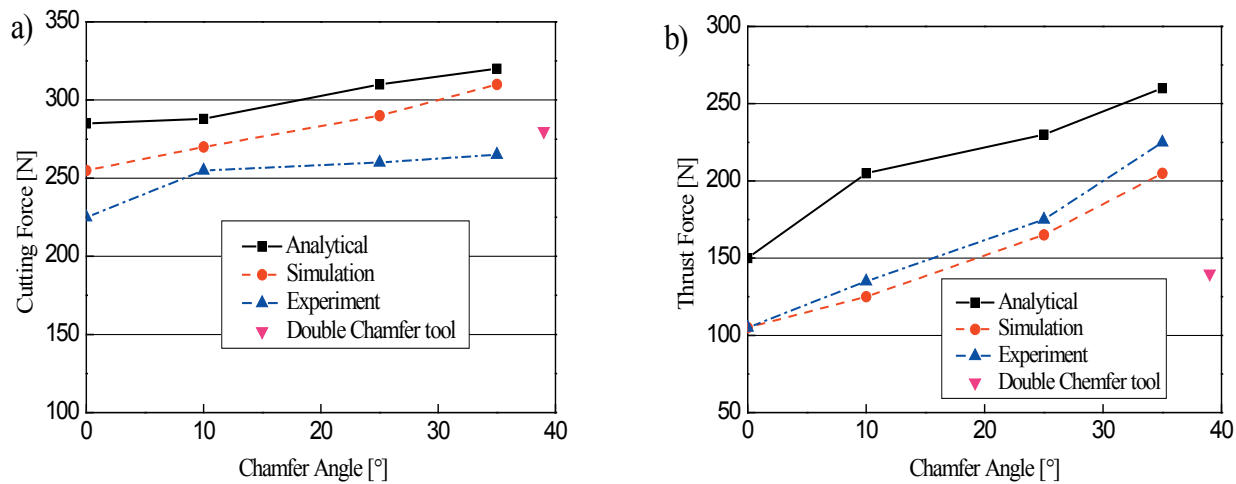

Fig. 13. Comparison of a) cutting and b) thrust forces between analytical, experimental and simulation results of tools with different chamfer angles, and simulation results of double chamfer tool 

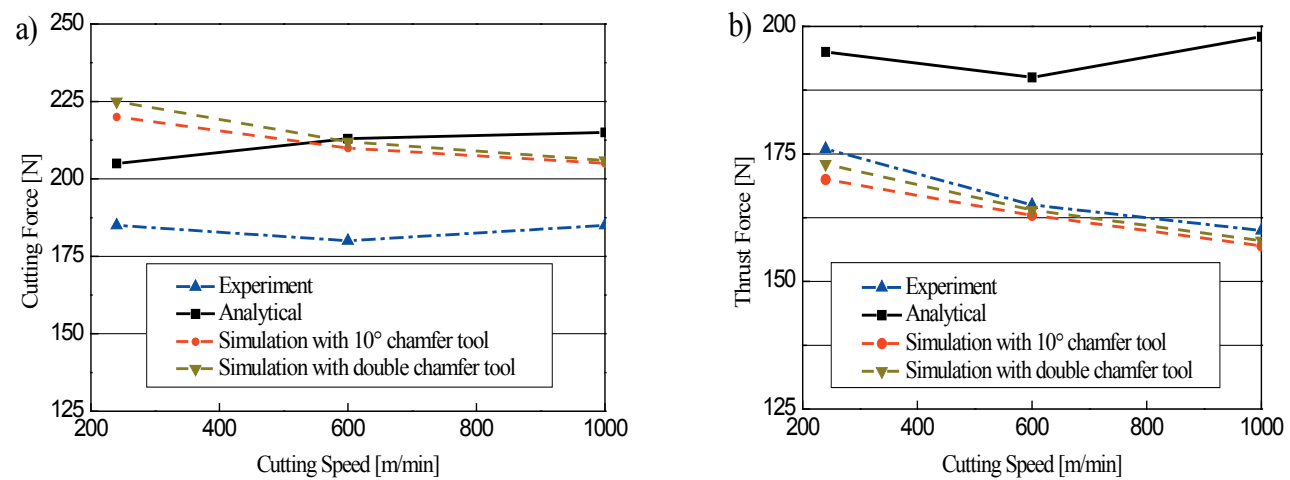

Fig. 14. Comparison of a) cutting and b) thrust forces between analytical, experimental and simulation results of $-10^{\circ}$ chamfer tool and simulation with double chamfer tool under different cutting speeds

that of increasing the hone radius. It can seen in Fig. 12 that the profiles of chamfer and double chamfer tool are almost same, but the peaks of them are both lower than the hone one. The peaks of the residual stresses obtained with the sharp tool are lowest among the other three tools, suggesting that the finer surface quality is acquired. Meanwhile, the experimental results from the operation with a $-25^{\circ}$ chamfer tool suggest that there is a little diference between measurment and prediction at the surface, which is partially due to the preexisting residual stresses on the actual machined surface that are neglected in the finite element model and the meshing quality in the simulations.

\subsection{Effects of the Tool Geometry and Cutting Speed on Machining Forces}

Fig. 13 shows the effect of different tool geometries (sharp, chamfer, double chamfer) on cutting forces and the thrust forces obtained by simulations and experiments. It can be seen that the thrust force acquired with the $-35^{\circ}$ chamfer angle is much larger than other four forces, since the presence of a chamfer angle leads to the buildup of the dead metal zone where the workpiece material will be strained to a larger extent, compared with the sharp tool. Moreover, the force created by the double chamfer tool is between that of the tools with $-10^{\circ}$ and $-25^{\circ}$ chamfer angles, because the region of the dead zone created by the double chamfer is moderate. Without considering the beginning of the cutting process when the tool has not been loaded, the cutting force gradually advances and up to a relatively large point, then fluctuates in a small area; the thrust force has the same trend but changes more rapidly. The analytical results obtained from the Ren and Altintas [21] show a great difference between the experimental and simulation results, but give a clear description of the trends of forces which is in qualitative agreement with the others. Nalbant et al. [2] concluded that it is an increment-decrement relationship between cutting speed and min cutting force. Increasing cutting speed by $66.6 \%$ (150 to $250 \mathrm{~m} / \mathrm{min})$ and $20 \%$ causes the main cutting force to decrease by 14.6 and $10.4 \%$, respectively. This trend can be also found in Fig. 14, showing that the machining forces decrease as the cutting speed increases. Moreover, the cutting speed has more significant influence on the thrust force in contrast with the cutting force. Meanwhile, the tool edge geometry has little effect on the machining force as the cutting velocity reaches a comparatively high value.

\section{CONCLUSIONS}

A finite element model for the simulation of chip formation with different cutting edge geometries during the cutting process has been presented. The simulation results show that the edge geometries affect the chip removal process less significantly, because the dead zones are formed under the chamfer, double chamfer and blunt edge to act as the main cutting edge of the tools, just like the sharp tool. The coupled thermal-mechanical analysis was conducted with four different tools, and some results were obtained. The behaviour and shape of the dead metal zone are studied by examining the distributions of the material velocity and the stress. The effect of tool edge geometry on the residual distribution was also studied, showing that the chamfer and double chamfer tool had the almost same function on the residual stress distribution in comparison with the hone tool and sharp tool. It is clear that the force in 
the thrust direction has more dependence on the tool geometry and cutting speed, in comparison with the one in the cutting direction. The forces increase as the chamfer angle increases, and decrease as the cutting speed increases. The cutting edge geometry has little influence on the machining force when the cutting speed reaches a very high level.

Finally, the present fulfillment of the ALE method is only applied to continuous chip formation, and can not directly extend to segmented chips, which need a better crack propagation scheme. A more complex mesh motion scheme should be used to allowed for the formation of new surfaces in chips.

\section{ACKNOWLEDGEMENTS}

The authors would like to thank Shanghai University of Engineering Science (Project Code: 14KY0107) for providing financial support for the project, and the reviewers for their suggestions.

\section{REFERENCES}

[1] Dogra, M., Sharma, V.S., Dureja, J. (2011). Effect of tool geometry variation on finish turning-A Review. Journal of Engineering Science and Technology Review, vol. 4, no. 1, p. 1-13.

[2] Nalbant, M., Altın, A., Gökkaya, H.. (2007). The effect of cutting speed and cutting tool geometry on machinability properties of nickel-base Inconel 718 super alloys. Materials \& Design, vol. 28, no. 4, p. 1334-1338, D0I:10.1016/j.matdes.2005.12.008.

[3] El-Wardany, T.I., Kishawy, H.A., Elbestawi, M. A.. (2000). Surface integrity of die material in high speed hard machining, Part 1: Micrographical analysis. Journal of Manufacturing Science and Engineering, vol. 122, no. 4, p. 620-631, DOI:10.1115/1.1286367.

[4] Shintani, K., Ueki, M., Fujimura, Y. (1989). Optimum cutting tool geometry when interrupted cutting carburized steel by CBN tool. International Journal of Machine Tools and Manufacture, vol. 29, no. 3, p. 415-423, D0l:10.1016/08906955(89)90010-2.

[5] Shintani, K., Ueki, M., Fujimura, Y. (1989). Optimum tool geometry of CBN tool for continuous turning of carburized steel. International Journal of Machine Tools and Manufacture, vol. 29, no. 3, p. 403-413, D0l:10.1016/08906955(89)90009-6.

[6] Matsumoto, Y., Hashimoto, F., Lahoti, G. (1999). Surface integrity generated by precision hard turning. CIRP Annals - Manufacturing Technology, vol. 48, no. 1, p. 59-62, D0I:10.1016/S0007-8506(07)63131-X.

[7] Kountanya, R., Al-Zkeri, I., Altan, T. (2009). Effect of tool edge geometry and cutting conditions on experimental and simulated chip morphology in orthogonal hard turning of $100 \mathrm{Cr} 6$ steel. Journal of Materials Processing Technology, vol. 209, no. 11, p. 5068-5076, D0l:10.1016/j. jmatprotec.2009.02.011.
[8] Shatla, M., Yen, Y.C., Altan, T. (2000). Tool-workpiece interface in orthogonal cutting-application of FEM modeling. Transactions of the North American Manufacturing Research Institution of SME, p. 173-178.

[9] Kim, K.W., Sins, H.-C. (1996). Development of a thermoviscoplastic cutting model using finite element method. International Journal of Machine Tools and Manufacture, vol. 36, no. 3, p. 379-397, Dol:10.1016/0890-6955(95)00054-2.

[10] Shi, G., Deng, X., Shet, C. (2002). A finite element study of the effect of friction in orthogonal metal cutting. Finite Elements in Analysis and Design, vol. 38, no. 9, p. 863-883, D0l:10.1016/ S0168-874X(01)00110-X.

[11] Movahhedy, M. R. (2000). ALE Simulation of Chip Formation in Orthogonal Metal Cutting Process, PhD thesis, University of British Columbia, Vancouver, p. 33-38.

[12] Waldorf, D.J., DeVor, R.E., Kapoor, S. G. (1999). An evaluation of ploughing models for orthogonal machining. Journal of Manufacturing Science and Engineering, vol. 121, no. 4, p. 550-558, DOI:10.1115/1.2833050.

[13] Al-Athel, K.S., Gadala, M.S. (2011). The use of volume of solid (VOS) approach in simulating metal cutting with chamfered and blunt tools. International Journal of Mechanical Sciences, vol. 53, no. 1, p. 23-30, D0l:10.1016/j.jijmecsci.2010.10.003.

[14] Jacobson, S., Wallén, P. (1988). A new classification system for dead zones in metal cutting. International Journal of Machine Tools and Manufacture, vol. 28, no. 4, p. 529-538, DOI:10.1016/0890-6955(88)90065-X.

[15] Liu, K., Melkote, S.N. (2007). Finite element analysis of the influence of tool edge radius on size effect in orthogonal micro-cutting process. International Journal of Mechanical Sciences, vol. 49, no. 5, p. 650-660, Dol:10.1016/j. ijmecsci.2006.09.012.

[16] Movahhedy, M.R., Altintas, Y., Gadala, M.S., (2002). Numerical analysis of metal cutting with chamfered and blunt tools. Journal of Manufacturing Science and Engineering, vol. 124, no. 2, p. 178-188, Dol:10.1115/1.1445147.

[17] Mu-oz-Sánchez, A., Canteli, J.A., Cantero, J.L., Miguélez, M.H.. (2011). Numerical analysis of the tool wear effect in the machining induced residual stresses. Simulation Modelling Practice and Theory, vol. 19, no. 2, p. 872-886, D0l:10.1016/j. simpat.2010.11.011.

[18] Johnson, G.R., Cook, W.H. (1983). A Constitutive Model and Data for Metals Subjected to Large Strains, High Strain Rates and High Temperatures, p. 541-547.

[19] Alvarez, R., Domingo, R., Sebastian, M.A. (2011). The formation of saw toothed chip in a titanium alloy: influence of constitutive models. Strojniški vestnik - Journal of Mechanical Engineering, vol. 57, no. 10, p. 739-749, Dol:10.5545/svjme.2011.106.

[20] Liu, M., Takagi, J.-i., Tsukuda, A. (2004). Effect of tool nose radius and tool wear on residual stress distribution in hard turning of bearing steel. Journal of Materials Processing Technology, vol. 150, no. 3, p. 234-241, D0l:10.1016/j. jmatprotec.2004.02.038.

[21] Ren, H., Altintas, Y., (2000). Mechanics of machining with chamfered tools. Journal of Manufacturing Science \& Engineering, vol. 122, no. 4, p. 650, Dol:10.1115/1.1286368. 